(1) B $O$ Observatori de

Bioètica i Dret

ISSN: 1886-5887

\section{Revista de Bioética y Derecho}

Publicación del Máster en Bioética y Derecho nww.bioeticayderecho.ub.edu

ARTíCULO

\title{
¿Hay lugar para el consentimiento informado en los tratamientos de las personas con psicosis? Una reflexión sobre el tratamiento de las psicosis
}

\author{
Is there place for informed consent in treatment of \\ psychotic patients? A reflection on treatments of psychosis
}

Miguel A. VALVerde *

JosÉ A. INCHAUSPE *

\footnotetext{
* Miguel A. Valverde. Psicólogo Clínico. Unidad de Hospitalización Psiquiátrica Sección A. Complejo Hospitalario de Pamplona, Osasunbidea, Navarra. mvalverde@cfnavarra.es

* José A. Inchauspe. Psiquiatra. Director Centro de Salud Mental San Juan, Osasunbidea, Pamplona, Navarra. ja.inchauspe.arostegui@cfnavarra.es
} 


\section{Índice}

1. El consentimiento informado en salud mental y la psicosis.

2. Dos tradiciones en Salud Mental.

3. Razones para soslayar el consentimiento informado en los pacientes psicóticos.

4. La clínica con pacientes psicóticos.

5. La vida bajo psicofármacos. El tratamiento correcto según la propuesta biologicista y sus resultados.

6. Las psicoterapias.

7. La perspectiva de los usuarios.

8. La red asistencial y el consentimiento informado.

9. Conclusiones.

\section{Resumen}

La práctica del consentimiento informado $(\mathrm{Cl})$ en Salud Mental es inhabitual, más aún para los pacientes psicóticos, a pesar de que tanto la legislación vigente, como las declaraciones de asociaciones profesionales, organizaciones de pacientes y códigos deontológicos defienden el consentimiento informado y la autonomía del paciente, también para las personas con psicosis. Es habitual que pacientes y allegados desconozcan el alcance, límites y riesgos del tratamiento farmacológico usual o la existencia de otros abordajes

Las razones del déficit de $\mathrm{Cl}$ pueden hallarse en las peculiaridades de estos pacientes, pero también en el presupuesto de que la psicosis impacta en el mismo núcleo de la persona, y por ello se ve al afectado como incapaz de comprender y consentir.

Coexisten hoy dos estilos diferentes de acoger al paciente con dificultades de tipo psicótico: uno, la corriente principal que tiene como objetivo bloquear el proceso psicótico y revertirlo mediante el uso principal de antipsicóticos, y otro, que incluye diversos modelos que acogen a la persona psicótica con sus dificultades, que propone construir un marco de comprensión para sus experiencias. Los resultados no muestran superioridad del modelo dominante, más bien todo lo contrario.

Cuando se da la palabra a los usuarios estos defienden su autonomía y derecho a decidir, rechazando los modelos coercitivos.

El Cl, dar poder para decidir al paciente, además de un derecho es también un factor de recuperación para muchos pacientes.

Su puesta en práctica necesitaría cambios significativos en la formación de los profesionales y en la constitución de las actuales Redes Asistenciales de Salud Mental. 
Palabras clave: Consentimiento Informado (Cl); autonomía del paciente; psicosis; psicoterapia; movimiento de la recuperación; psicofármacos.

\section{Abstract}

Informed consent (IC) in Mental Health care is unusual, especially for psychotic patients. However, current legislation, statements of professional associations, patient organizations and deontological codes advocate informed consent and patients' autonomy, also for people with psychosis. Patients and their relatives usually are unaware of the scope, limits and risks of drug treatment and the existence of other approaches.

The reasons for the IC's lack can be found in the peculiarities of these patients, but also on the assumption that psychosis impacts the core of the person, and therefore they are unfit of understanding and consent.

Today there are two different ways to receive the patient with psychosis problems: first, the mainstream whose objective is to block the process and reverse psychotic symptoms principally using antipsychotics drugs, and another one, that includes various clinic models, that receive psychotic person with their difficulties, proposing to build a framework of understanding for their experiences. The outcomes don't reveal superiority for the mainstream model, rather the opposite.

When listening to the users, they clearly opt for their autonomy and right to choose, rejecting coercive models.

Informed consent is a right that empowers the patients and also a recovery factor for many of them.

Its implementation will require significant changes in the training of professionals and in the current Mental Health Care networks.

Key words: Informed Consent (IC); patient autonomy; psychosis; recovery movement; psychotropic drugs; psychotherapy. 


\section{El consentimiento informado en salud mental y la psicosis}

La falta de consentimiento informado $(\mathrm{Cl})$ en Salud Mental se observa de forma generalizada en la práctica clínica diaria. Los pacientes no saben que pueden esperar del tratamiento asignado, cómo decidir si ha tenido éxito o no, cuales son los efectos indeseados a corto y a largo plazo, y otras cuestiones relevantes (1-3). Las carencias en el consentimiento informado han sido objeto habitual de estudio y reclamación en salud mental (4-11). La demanda de respeto al principio de autonomía y el derecho a saber es objetivo principal de organizaciones de pacientes, como el movimiento por la recuperación o "recovery movement" (12-14).

Para los pacientes psicóticos la situación es especial, ya que suelen ser objeto de presiones coercitivas para medicarse o ingresar, a veces con intervención judicial, y rara vez se modifica el tratamiento cuando lo solicitan, incluso sí el estado psicótico ha remitido. Pocas veces conocen sus derechos para elegir o rechazar un tratamiento y como hacerlos valer (15-27). El tratamiento es elegido por el psiquiatra, y los pacientes y sus allegados no son informados de otras opciones clínicas.

\section{Dos tradiciones en Salud Mental}

En la asistencia actual conviven dos estilos que parten de tradiciones clínicas divergentes. La dominante enraíza con la obra de Kraepelin y la otra con el tratamiento moral de Pinel y Tukey en el siglo XIX.

Hoy la corriente dominante en Salud Mental se reconoce como una psiquiatría biologicista que da el valor máximo, muchas veces único, al tratamiento farmacológico del paciente psicótico, utilizando si fuera necesario métodos coercitivos.

La otra corriente considera que el paciente debe elegir aceptar o no un tratamiento, más aún si está fuera de la crisis psicótica aguda y no corre peligro, y entiende que su recuperación está imbricada con su autonomía y su capacidad de elegir (28). Esta orientación es compatible con lo que defienden las organizaciones de pacientes más importantes $(13,21,29-31)$, diversas escuelas clínicas de orientación psicosocial $(15,19,25,26)$, y algunas de otro tipo $(32,33)$.

De ambas líneas recogeremos argumentos sobre el consentimiento informado $(\mathrm{Cl})$ en la psicosis. No hay que suponer que todos los profesionales de determinada orientación comparten la misma perspectiva ética. Pero de sus presupuestos se pueden deducir argumentos a favor de una u otra opción respecto al valor de la autonomía del paciente psicótico. 


\section{Razones para soslayar el consentimiento informado en los pacientes psicóticos}

Hay argumentos que parecen sustentar la falta del consentimiento informado basándose en el concepto de la psicosis que defiende la psiquiatría biológica, aunque no explican por qué ocurre algo semejante en los demás pacientes.

Recordemos que en la literatura bioética (34-36) hay poco sobre cómo informar a personas con dificultades emocionales. Tales situaciones se resuelven consultando a los allegados. Parece sugerirse que estar emocionalmente afectado conlleva cierta incapacidad ante una decisión clínica.

No es fácil encontrar argumentos que defiendan que no deba hacerse uso del consentimiento informado en salud mental, incluso con personas psicóticas, aunque se acepte el tratamiento involuntario (37), ya que "a veces al no ser capaces de tomar una decisión racional, estas personas deben ser forzadas a conseguir un tratamiento aun en contra de sus deseos..." (38).

Las razones del déficit del $\mathrm{Cl}$ para el paciente psicótico pueden clasificarse en cuatro áreas:

a) Comunes al conjunto de pacientes de salud mental, que estando perturbados cognitiva y conductualmente se entiende que tienen dificultades para comprender y decidir.

b) Argumentos derivados de la práctica clínica del tipo: no se puede saber como evolucionará el cuadro clínico, no hay más tratamiento que el farmacológico, informar puede llevar a discusiones sin fin, aumentar su angustia y complicar el tratamiento, sin cierto nivel de coerción no se medicará y el proceso psicótico se reagudizará, etc. (23).

Medicar como opción imprescindible es una idea muy afianzada, y que el paciente se medique, aún en contra de su opinión, puede convertirse en objetivo clínico superior (37-39), como se constata en la mayoría de los manuales de psicopatología y psicofarmacología (40-43).

c) La tradición paternalista en Salud Mental.

Actuar para el paciente sin contar con él viene de antiguo, incluso hoy es una práctica común $(21,25)$. Esta actitud produjo actuaciones crueles, aunque en su tiempo se vieron como ciencia puntera, como la inoculación de la malaria o la lobotomía, procedimientos creados por Julius WagnerJauregg y Egas Moniz respectivamente, citando a los únicos psiquiatras con un premio Nobel. El principio de tales intervenciones, actuar sobre el cuerpo del paciente para cambiar su comportamiento, sigue vigente en la práctica dominante (5).

d) La psicosis entendida de forma tal en que es el propio núcleo del ser persona el que está colonizado por la enfermedad (37).

Se trata quizás del obstáculo más poderoso para la práctica del $\mathrm{Cl}$ en estos pacientes, ya que el psicótico tiene alteradas biológicamente sus facultades superiores estando enfermo en su propio "ser persona" $(37,44)$.

Esta concepción es reconocible en Kraepelin (1856-1926), quién recogía el habla de sus pacientes psicóticos de forma extensa y minuciosa, pero considerando sólo su perspectiva formal, sin 
interesarse por su significado y su sentido comunicativo (25). La corriente biológica actual, también denominada neokraepeliana, comparte esa actitud, y ha perdido interés en el psicótico como sujeto que relata sus experiencias y se comunica $(38,41)$.

Berrios califica el delirio psicótico como actos "lingüísticos vacíos", que no informan del sujeto, de sus preocupaciones o de su mundo interno. El delirio son palabras encadenadas al azar que han cristalizado de cierta forma como podrían haberlo hecho de otra (45). Intentar averiguar su sentido no debería entretener a los clínicos $(41,42)$, ya que el delirio solo constata y refleja la enfermedad cerebral del paciente (46).

Andreasen, icono de esta psiquiatría biológica, describe una práctica clínica ideal en estos términos: "Cuando las visitas de 15 minutos reemplacen a las de 50 minutos estos [los pacientes] las preferirán (...) Las visitas más cortas son una forma más eficiente y económica para tratar a más personas. Muchas veces, 15 minutos son suficientes para que el médico y el paciente hablen de los síntomas y sobre como afectan a su vida" (38). La mayor parte de los clínicos, incluidos los biologicista, criticarían una práctica tan extrema (32). No obstante, reducir la clínica a preguntas sobre la acción de la medicación, soslayando el vivir y el sentido de las vivencias, es habitual en salud mental.

Considerar la psicosis desligada de la persona, es decir no conectada a los contextos personales, deshumaniza la psicosis (47) y hace que compartir las decisiones clínicas pueda parecer innecesario.

Un concepto clave para esta perspectiva es la falta de conciencia de enfermedad. Se afirma que el psicótico no puede verse a sí mismo como enfermo que necesita tratamiento, ya que la psicosis se caracteriza frecuentemente por su "falta de conciencia de enfermedad". De esta forma el desacuerdo con el terapeuta y un eventual rechazo de la medicación es interpretado por el clínico como un síntoma más, dentro de la constelación de síntomas característicos de la psicosis $(37,47-$ 49).

Desde este modelo se fomenta la conciencia de enfermedad, como parte del tratamiento, introduciendo en el paciente la idea de que sufre un trastorno cerebral crónico $(37,42,46)$. Se trasmite un relato que sitúa al paciente como enfermo, desligado de su biografía y de sus vivencias, eliminando de la psicosis la subjetividad. La resistencia del paciente a aceptar esta visión se considera parte de su enfermedad.

Muchos expertos no aceptan que el estado psicótico sea producto de una alteración cerebral (25,50-52), a lo que se ha replicado que pueden estar presos de su propia ignorancia, como escribió Fuller Torrey (53) de los autores de la obra Modelos de Locura (26). 


\section{La clínica con pacientes psicóticos}

Los argumentos de los puntos a) y b) del capítulo anterior no son banales. Constatan que la relación con el paciente psicótico puede ser complicada.

Al modelo conceptual de la psicosis colonizadora se le oponen pruebas de investigación y clínicas $(25,51,54,55)$.

Los estudios referidos a la competencia para decidir del paciente psicótico muestran resultados ambiguos, pero nunca una incapacidad generalizada en todos ellos, ni durante todo su proceso. Amador encontró incompetencia asociada a falta de conciencia de enfermedad en un $60 \%$ de los casos (48). Un estudio que evaluaba la competencia del paciente psicótico encontró que hasta el $48 \%$ tenía capacidad de consentir, medida mediante la escala MacCAT, pero otros estudios no muestran diferencias claras en comprensión respecto a la población control, otros indican que la capacidad de comprender y consentir no está relacionada con la sintomatología psicótica, y otros resultan ambiguos (56). Sea como fuere el paciente psicótico no suele ser informado $(7,57)$.

Desde la práctica clínica lo primero a considerar es que existen dos situaciones diferentes, la de la crisis aguda y fuera de ella. La primera puede ser una situación de urgencia en la que peligra la imagen social del paciente, puede conllevar comportamientos disruptivos, estados emocionales intensos, o riesgo de auto y/o heteroagresión.

Conviene recordar que es posible entenderse con las personas en dificultades. Se necesitan para ello ciertas habilidades que pueden ser aprendidas y ensayadas, mediante formación específica Posiblemente pocos profesionales conseguirán comunicarse bien en todos los casos y situaciones, pero si podrán hacerlo, con un estilo relacional adecuado y una estrategia correcta, en buena parte de los mismos. Es un tipo de formación que parece descuidarse hoy en España en el programa MIR, PIR, etc.

La forma habitual de manejo de la crisis es bloquear la sintomatología psicótica con medicación, y contener y aislar al paciente si es intensa. Después de la crisis, el modelo biológico actual propone un tratamiento de mantenimiento a medio y largo plazo con antipsicóticos $(58,59)$, combinados habitualmente con otros psicofármacos (60-62). Existe un abanico de recursos psicosociales poco implantados, con resultados contrastados, utilizados en general como apoyo a la estrategia farmacológica, incluso en modelos de uso intensivo (63).

Incluso dentro de esta práctica podría el $\mathrm{Cl}$ tener mayor lugar que el actual porque fuera de la crisis, y si el paciente siguiera sin ser capaz de elegir, sus allegados sí podrían hacerlo, y en algún momento, también el paciente estará capacitado para elegir, si el clínico comparte su tiempo con él y muestra comprensión, porque el paciente psicótico no es incapaz todo el tiempo, ni para todas las cosas.

Existe otra orientación asistencial que acoge al paciente psicótico como persona en dificultades, que practica una asistencia colaborativa y conlleva unos objetivos clínicos distintos, compartidos y que no se reducen a la desaparición de la sintomatología. Desde esta perspectiva, el 
principio de tratar a otros del modo en que nos gustaría ser tratados nosotros mismos es la base ética para defender el consentimiento informado y considerar la autonomía del paciente psicótico.

Es un estilo que enraíza con el tratamiento moral de Pinel y Tuke en el s XIX, y es reconocible en diversas orientaciones terapéuticas, incluyendo las psicoterapias recientes $(26,64)$.

La persona en una crisis psicótica puede estar descentrada u ocupada en cuestiones que interfieren la gestión de su vida, y no considerar adecuadamente sus dificultades. En cambio sí suele tener conciencia de dificultades. Acoger al paciente con sus dificultades subjetivas, con apoyo y colaboración, promoviendo un espacio para la comprensión de sus experiencias es otra forma de asistir.

Es un estilo común a diversos abordajes terapéuticos, incluyendo al movimiento de recuperación, gestionado por los propios pacientes. Sobre esta perspectiva comprensiva, que es también una forma de relación con la persona psicótica, mediante la aceptación y el reconocimiento personal, puede desarrollarse un relato en el que el paciente juega un papel protagonista que le oriente más allá del trastorno o enfermedad, hacia una vida con sentido. La psicosis, se entiende aquí, no es un mero desvarío. El respeto a su proceso, a su tiempo y sus necesidades, es clave para acoger y encontrarse con la persona en dificultades.

En otras palabras, la práctica del $\mathrm{Cl}$, además de un imperativo ético, forma parte de la propia naturaleza de este tipo de intervenciones clínicas.

\section{La vida bajo psicofármacos. El tratamiento correcto según la propuesta biologicista y sus resultados}

La corriente biologicista aboga por intervenir en la psicosis para combatir sus síntomas, bloquear e idealmente revertir un hipotético proceso biológico subyacente mediante el uso de antipsicóticos, normalmente a largo plazo. Para ello necesita fomentar la conciencia de enfermedad en el paciente y sus allegados. El denominador común de todos los dispositivos de la red asistencial por los que el paciente transite será asegurar en primer lugar la toma de medicación (65). A los allegados se les transmitirá ese mismo mensaje (66). Para cumplir con la toma de medicación y combatir la tendencia bien conocida del paciente a abandonarla (37,67-69), se utilizarán medios más o menos coercitivos, que pueden llegar a la autorización judicial para realizar tratamientos forzosos (24).

Proponer un tratamiento de mantenimiento con antipsicóticos a medio y largo plazo es relativamente reciente y no era la práctica de hace 30 años, décadas después de su introducción, cuando se proponía la retirada del fármaco después de remitir los síntomas, para volver a instaurarlos si reaparecían $(70,71)$. Hoy en día se entiende que la interrupción de la medicación antipsicótica tras un primer episodio psicótico aumenta considerablemente el riesgo de recaída a corto plazo $(58,59)$, y que en pacientes con varios episodios el tratamiento de mantenimiento está asociado a una menor 
frecuencia de recaídas $(5,65)$, que la disminución progresiva o interrupción del fármaco tras la recuperación del primer episodio (58).

Aunque así fuera, el precio a pagar por disminuir el riesgo de recaídas mediante un tratamiento de mantenimiento con antipsicóticos es alto.

Recordemos sus efectos adversos a largo plazo sobre el sistema cardiovascular (72), el metabólico en el que asistimos hoy en día a una auténtica epidemia de diabetes tipo II, dislipemias y obesidad entre pacientes que toman antipsicóticos de nueva generación (124), el neurológico, con déficits en atención, memoria, disfrute, motivación y activación, y la pérdida de masa cerebral progresiva, dependiendo de la dosis de antipsicóticos y su combinación con otros psicofármacos (64, 73-76)

Su uso fomenta estilos insanos, como la sedentariedad y el consumo de grandes cantidades de comida, tabaco, café, alcohol o cánnabis, quizás para compensar el malestar inducido por el tratamiento, y mala calidad de vida, que puede desembocar en aislamiento social y suicidios, algo que según algunos autores no siempre fue así para los pacientes con psicosis $(6,77)$. Se estima que usar antipsicóticos a largo plazo puede hacer perder 20 años o más de vida $(6,79,80)$.

No suele informarse a pacientes y familiares de los riesgos del tratamiento farmacológico, aunque se les informa enfáticamente de la gravedad del trastorno.

Este modelo fomenta que el paciente se vea a sí mismo como enfermo crónico, estigmatizado al ser tratado como incapaz de regirse, y forzado a tomar fármacos desagradables que le abotargan emocionalmente. Tendrá que aceptar una vida con escasas perspectivas de futuro, pero si lo hace se dirá que tiene una adecuada "conciencia de enfermedad". Lo frecuente es que el paciente intente huir de tal perspectiva.

Muchos de estos pacientes en crisis psicótica o fuera de ella, intentarán deshacerse del tratamiento farmacológico $(21,67-69)$ corriendo el riesgo de una recaída a corto plazo $(1,5)$. Aun aceptando el carácter preventivo de recaídas de la medicación antipsicótica a corto plazo existen otros factores explicativos de la recaída. Si dejan la medicación se encontrarán subjetivamente mejor, pero al ser "descubiertos" se redoblará el control sobre ellos, y el mensaje "estás enfermo" se intensificará en un contexto de tensión ambiental. Podrán ser víctimas del síndrome de discontinuación al abandonar bruscamente los antipsicóticos, sin monitorización y asesoramiento clínico, e incluso podrán ser reingresados por dejar la medicación, sin recaída propiamente dicha. En un entorno tenso, exasperante, controlador, con mensajes de incapacidad, y otros del tipo "es por tu bien", el paciente se verá dentro de una marejada relacional, bajo los efectos de más fármacos y con la etiqueta añadida de paciente difícil, en un estado de indefensión y desesperanza $(15,21,20,26)$.

En consecuencia este modelo asistencial no solo fracasa frecuentemente a la hora de establecer una alianza terapéutica con el paciente sino que en ocasiones se posiciona en lucha con sus pacientes, que pueden llegar a temer a sus sanadores más que a sus dificultades previas (81). No es extraño que los estudios muestren mejor recuperación para los pacientes psicóticos que tienen un entorno que les presta poca o ninguna atención $(25,26,49)$. 
Históricamente se ha constatado que algunos procesos psicóticos cursaban hacia la recuperación por si solos, hasta en la tercera parte de los casos (82). Pero quizás hoy ya no sea así y el porcentaje de pacientes que se recuperan sea menor.

Sucede que la asistencia actual obtiene resultados muy pobres en cuanto a recuperación funcional, siendo la incapacidad laboral y un funcionamiento personal y social empobrecido el destino habitual para muchos pacientes $(83,84)$. Se ha sugerido que estos resultados son peores que en el pasado (Ver Figura 1, que muestra el porcentaje de pacientes mejorados, con un criterio relativamente exigente, con síntomas disminuidos, y capacidad para vivir en comunidad) (85).

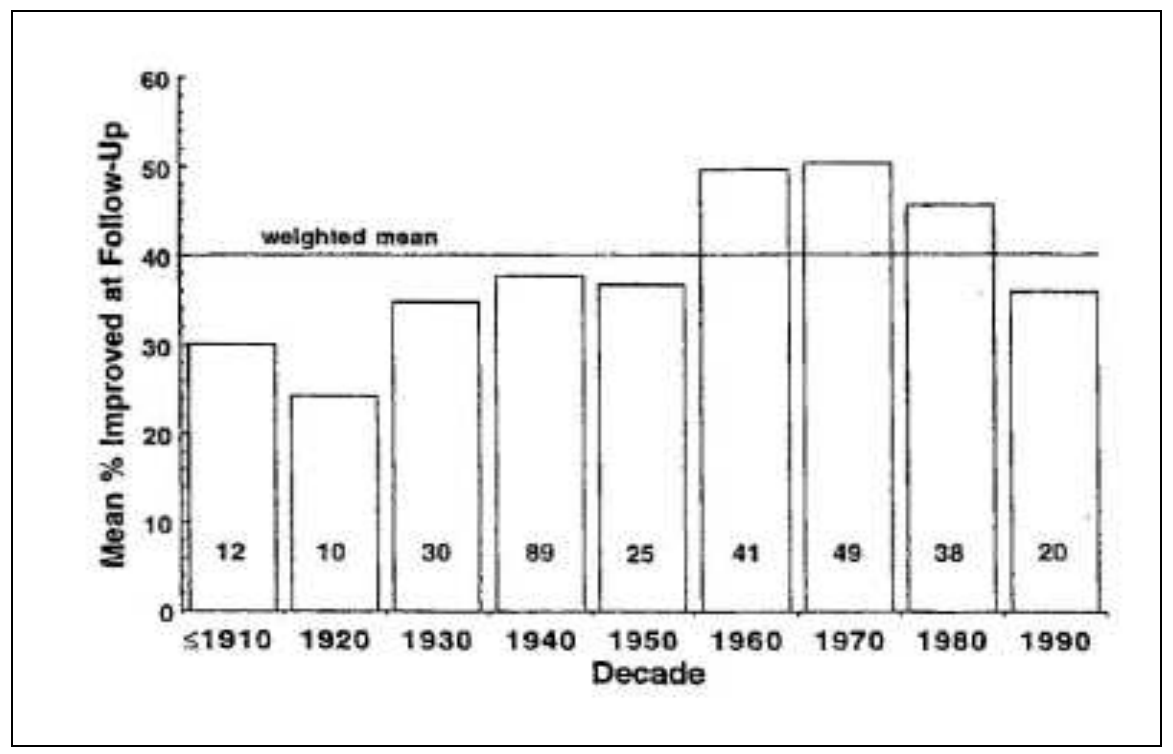

Figura 1. Porcentaje de pacientes considerados mejorados, en seguimientos de 10 años o más, 314 cohortes, por décadas, en el S XX El número dentro de las columnas indica el número de cohortes estudiadas en esa década. La línea que cruza horizontalmente el gráfico es la media de pacientes en mejoría considerando todas las cohortes. Hegarty \& al 1994 (80).

Los antipsicóticos producen mejoría significativa para un porcentaje de pacientes pequeño (un 20-30\%). Para otros la mejoría solo es apreciable en puntuaciones en escalas como la PANSS, pudiendo no ser clínicamente significativa (19). Finalmente para cerca del $40 \%$, no hay mejoría, quizás empeoramiento, a veces progresivo y rápido (5). Los efectos adversos han sido minimizados y pueden ser graves e impedir la recuperación $(19,73-75,86)$. No hay pruebas de eficacia en el mantenimiento con medicación antipsicótica a largo plazo, sino más bien de lo contrario $(5,83,87)$.

El estudio de seguimiento de Chicago, Chicago Follow-Up Study, que ha presentado los resultados a 20 años, observó la evolución de los pacientes desde su debut, muestra, entre otros aspectos, la recuperación entre quienes siguieron el tratamiento habitual (tomar antipsicóticos en una perspectiva a largo plazo) y quienes dejaron los fármacos. $Y$ en cierto sentido permite analizar diferentes trayectorias. A los dos años quienes no tomaban fármacos estaban en mejor estado, pero a los cinco años la diferencia en aspectos funcionales era muy amplia. En las Figuras 2 y 3 observamos la diferencia de resultados a los 15 años, que muestra que apenas existe recuperación entre quienes permanecen con antipsicóticos. Señalemos que la definición de recuperación es más exigente que la considerada en la Figura 1, ya que considera un episodio de recuperación a permanece sin síntomas e ingresos, mantener actividad laboral, y vida social, durante un año (83). A los 20 años de seguimiento, este estudio constata que a quienes dejaron la medicación, lo hicieron 
"por decisión propia, a veces en contra la indicación profesional" les va "relativamente bien, o mejor que quienes tomaron medicación de forma continua" (88).

La opción de no tratarse a largo plazo, aunque tenga riesgos, puede no ser incorrecta para bastantes pacientes, ya que hasta el $40 \%$ obtiene periodos de recuperación.

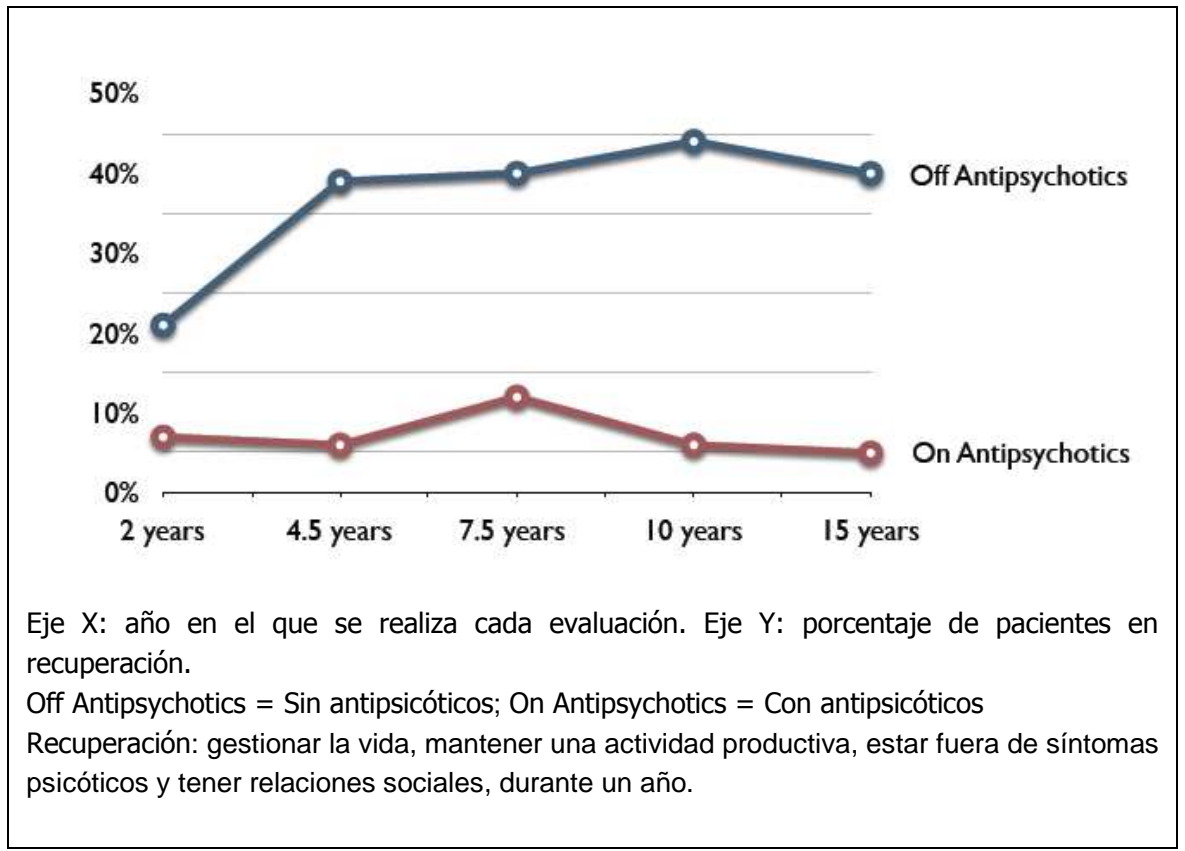

Figura 2: Tasa de recuperación a largo plazo en pacientes con esquizofrenia (Harrow \& Jobe 2007 (83)).

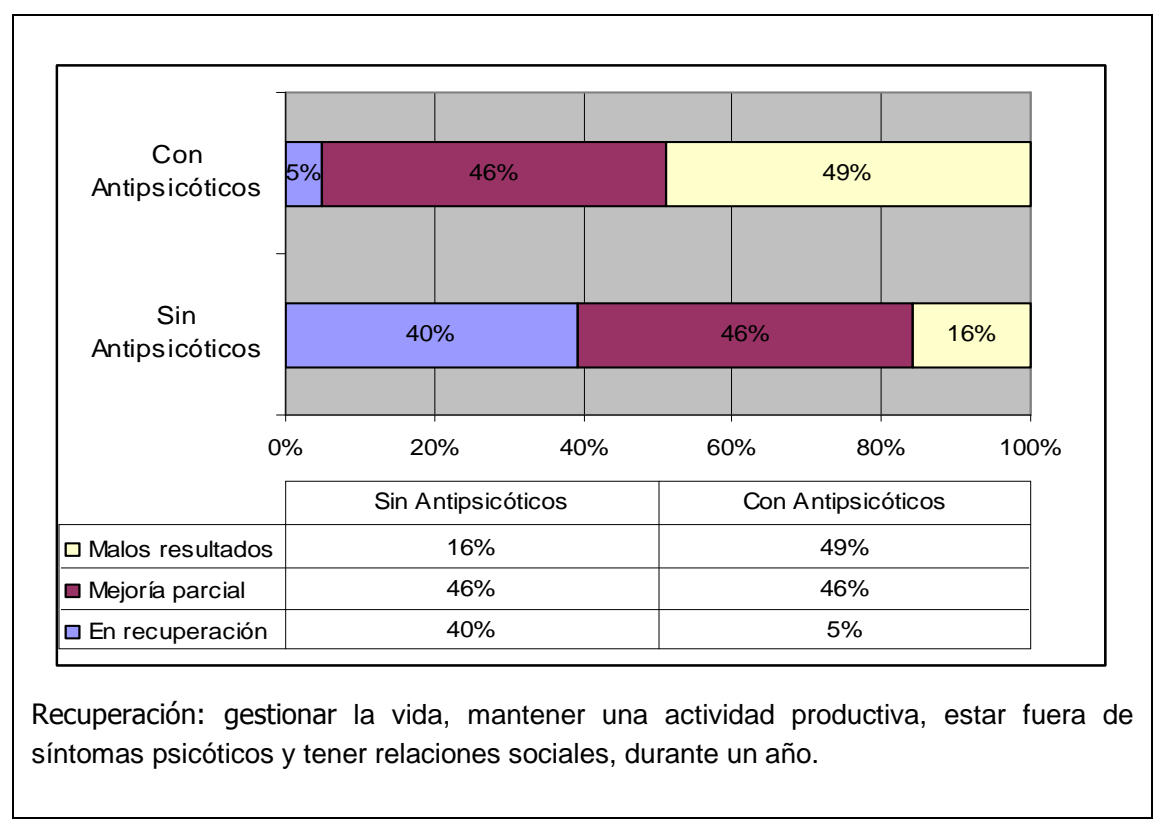

Figura 3: Estado de los pacientes con esquizofrenia a los 15 años de seguimiento (Harrow \& Jobe 2007 (83)).

La psiquiatría biologicista actual es particularmente impermeable a los resultados e investigaciones de otras orientaciones clínicas. Fundamenta su medicina basada en la evidencia en ensayos clínicos aleatorizados, ECAs, a corto plazo, muy alejados de la práctica clínica naturalística, dándose como objetivo la neutralización de los síntomas y perdiendo de vista la recuperación funcional (59). Además, su práctica está influenciada por la información propagada por líderes de 
opinión de la profesión, “key opinion leaders”, usualmente ligados a la industria farmacéutica $(6,89$, 90).

Solo muy recientemente la corriente biologicista se está haciendo eco de la potencial severidad de los efectos adversos a largo plazo de los antipsicóticos $(73,86)$ y la escasa efectividad de las estrategias de mantenimiento a largo plazo $(58,83)$ con la publicación en revistas de prestigio de editoriales con títulos tan expresivos como "Antipsicóticos... el primer episodio de la psicosis: menos es más" (59), y se llega a reflexiones del tipo "Antipsicóticos: ¿es hora de considerar la elección del paciente?" (19) o "El futuro de la psiquiatría universitaria puede ser social" (129).

\section{Las psicoterapias}

Acoger al paciente psicótico considerando sus dificultades, incluyendo las cogniciones erradas, darle apoyo, colaborar para encontrar sentido a su experiencia psicótica y ayudarle a mejorar la vida buscando la recuperación funcional, es objeto de otro tipo de abordaje clínico en el que las psicoterapias ocupan un lugar central

Los abordajes psicoterapéuticos consideran los delirios y alucinaciones como experiencias personales con sentido (Cuadro 1), en las que también se puede hallar motivación para el cambio. La terapia Diálogo Abierto (55), las de orientación narrativa (91, 92), las que incluyen a la familia $(93,94)$, las de orientación dinámica (95), las terapias cognitivo conductuales (TCC), como la de Morrison (96), las TCC de tercera generación $(50,97)$, y otras intervenciones $(98,99)$, reconocen la experiencia psicótica en una perspectiva humana, y ayudan a integrar el delirio y las alucinaciones en los contextos personales (100-102). Según muchos autores, incluyendo a psiquiatras como Jim van Os, el abordaje psicoterapéutico podría ser la primera opción ante alucinaciones y delirios (103).

Se estima que la medicación con psicoterapia es mejor que sola, pero la psicoterapia sola, o a veces con fármacos durante breve tiempo y en pequeñas dosis, puede obtener mejores resultados (95), pudiendo ser más imprescindible la psicoterapia que los antipsicóticos para la recuperación (104).

\section{Acerca de las alucinaciones auditivas}

1. Escuchar voces es una experiencia humana normal, aunque no muy frecuente.

2. Pueden ser comprendidas en el contexto de las experiencias vitales y la narrativa personal, tienen un significado psicológico y un sentido.

3. Puede ser un fenómeno precipitado o intensificado por sucesos que superan y dejan indefensa a la persona. Pueden estar asociadas a episodios traumáticos. 
4. Las características estructurales de las voces son similares en la psicosis, en pacientes no psicóticos y en personas sin problemas de salud mental.

5. Lo vincula el escuchar voces con problemas de salud mental es la relación del sujeto con ellas, su reacción emocional.

Cuadro 1. Sobre las voces, según Hearing Voices Movement (Longden \& al 2012) (102).

Hay éxito en la psicosis para varios tipos de psicoterapia, aunque la mayor parte de su evaluación se ha realizado asociada al tratamiento con antipsicóticos, pero por suerte no toda $(55,87$, 95, 105-107, 128). Existen estudios que comparan a pacientes tratados con fármacos y sin ellos, especialmente antes de los años ochenta. Muestran, según numerosos autores, que en seguimientos a más de un año los pacientes sin fármacos se recuperan mejor $(2,5,87,95)$.

Actualmente mediante la terapia Diálogo Abierto, evitando internamientos y medicación en casi todos los casos, se consiguen las mejores tasas de recuperación, muy alejadas de las que se obtienen con medicación, ver Cuadro $2(87,100,108)$. Incluso las obtenidas por el tratamiento moral hace dos siglos resultan mejores $(64,87)$.

\section{Pacientes ( $\mathrm{N}=75)$}

\section{Esquizofrenia $(\mathrm{N}=30)$}

Otros trastornos psicóticos ( $\mathrm{N}=45)$

\begin{tabular}{l|l}
\hline Uso de Antipsicóticos & \\
\hline Nunca fueron expuestos & $\mid 67 \%$ \\
Uso ocasional durante los cinco años & $\mid 33 \%$ \\
Tomándolos al finalizar los cinco años & $\mid 20 \%$ \\
\hline Síntomas psicóticos & \\
\hline Nunca recayeron en cinco años & $\mid 67 \%$ \\
Asintomáticos a los cinco años & $\mid 79 \%$ \\
\hline Resultados a los cinco años & \\
\hline Trabajando o estudiando & $\mid 73 \%$ \\
Buscando trabajo & $\mid 7 \%$ \\
Discapacitados & $\mid 20 \%$
\end{tabular}

Cuadro 2: Resultados a los cinco años en todos los primeros episodios de psicosis en Laponia Occidental tratados con la Terapia Diálogo Abierto (Seikkula \& al 2006, (108)). 


\section{La perspectiva de los usuarios}

En nuestro país hay pocas experiencias asociativas protagonizadas por usuarios con el perfil del movimiento de la recuperación, aunque existen asociaciones de familiares que ejercen de grupo de presión ante la administración y facilitan apoyo y servicios de tipo socioeducativo, en general muy en sintonía con la corriente dominante de la asistencia actual. Aun con todo hay algunas experiencias en los que los usuarios muestran sus vivencias, como Radio Nicosia, primeravocal.org, soyborderline.com, o el programa La Ventana en la Cadena SER.

En el Reino Unido, USA, Holanda, Brasil y Australia hay pacientes, muchos diagnosticados de trastorno mental grave, que se han asociado para intervenir en aspectos asistenciales y de investigación. Este movimiento, denominado de la recuperación (Recovery Movement), se ha hecho oír ante profesionales de la Salud Mental y la sociedad en general construyendo un discurso de denuncia respecto a no haber sido tratados adecuadamente, ocultándoseles información y sin considerar su palabra, discurso actualmente recogido y apoyado por numerosos profesionales.

Las reuniones de autoayuda del movimiento de la recuperación están influenciadas por la obra de Romme \& Escher "Dando sentido a las voces" (101, 109), y consideran la sintomatología psicótica como experiencias con sentido (ver Cuadro 1) $(109,110)$. Promueven una narrativa personal para trascender a una nueva identidad, más allá de la enfermedad psicótica. El grupo de autoayuda quiere ser un espacio seguro, que facilita apoyo e inspiración. Un testimonio refiere que dentro del grupo "puedo hablar de las alucinaciones y la depresión sin miedo a ser juzgado, o a que se me aumente la medicación o a ser ingresado" (111).

Su trabajo está avalado por una ingente cantidad de relatos personales, que refieren recuperación $(16,81,112,113)$. Profesionales como Williams (114) y Warner (115), entre otros, consideran que la ayuda prestada por el movimiento de recuperación es válida $(16,81,113,116)$. Williams indica que hay cinco factores claves de la recuperación que comparte este movimiento: a) Esperanza en que la recuperación es posible, b) Comprender la psicosis como una experiencia personal, no como una enfermedad cerebral, c) Encontrar sentido a las experiencias psicóticas, d) Conectar con la vitalidad de la persona, e) Implicarse en relaciones sociales y comunitarias (117). Estos grupos de autoayuda, y las psicoterapias, comparten estos supuestos, y seguramente no es extraño que obtengan resultados positivos: la escucha, el apoyo compasivo, y la creación de un espacio para la esperanza pueden ser mejores que un modelo de enfermedad centrado en el uso más o menos coercitivo de fármacos que provocan malestar y entorpecen la regulación emocional ( 2 , $20,64,118,119)$.

El movimiento de la recuperación postula que los usuarios tienen mucho que decir sobre los tratamientos recibidos, que pueden juzgar su eficacia, que pueden opinar sobre los servicios asistenciales, y además realizar investigación al respecto $(21,81)$. 
Todos los seres humanos son creados diferentes.

Todo ser humano tiene derecho a ser mentalmente libre e independiente.

Todo ser humano tiene derecho a sentir, ver, imaginar, intuir, creer o experimentar, de cualquier manera y en cualquier momento.

Todo ser humano tiene derecho a comportarse de cualquier manera que no dañe a otros ni viole leyes justas.

Ningún ser humano será sometido sin su consentimiento a reclusión, encierro, castigo o intervenciones médicas o psicológicas destinadas a intentar alterar sus pensamientos, sentimientos o experiencias.

Cuadro 3: Declaración Universal de los Derechos y Libertades Mentales, realizado por el grupo Mad Pride, dentro del movimiento de la recuperación (120).

No es en puridad un movimiento antifármacos, pero pretende hacer valer el derecho a elegir si tomarlos o no, a ser informados de sus efectos, de su utilidad y de la existencia de tratamientos alternativos (21)

Respecto al tratamiento emergen dos cuestiones claves. Una es que algunos pacientes psicóticos cuando se encuentran mal son conscientes de necesitar ayuda, la intentan conseguir, pero también intentan alejarse de donde se les interne o se les medique obligatoriamente (121). La otra es que cuando los pacientes solicitan reducir su medicación no suelen recibir apoyo profesional, es decir no se acepta su autonomía (21). La declaración de los derechos mentales surgida en este movimiento tiene que ver con "protegerse" de la asistencia recibida sin su consentimiento (Cuadro 3 ).

\section{La red asistencial y el consentimiento informado.}

La práctica del $\mathrm{Cl}$ puede chocar con la actitud clínica de la asistencia psiquiátrica actual y su estrategia centrada en bloquear los síntomas psicóticos mediante fármacos como objetivo prioritario. Es posible que hasta que no se considere acoger al paciente psicótico como persona en dificultades, en vez de enfermo incapacitado en ser persona, no haya cambios sustanciales en el Cl. Incluso puede que no se tome conciencia de que parte de los pacientes psicóticos temen la ayuda que se les presta.

De cualquier modo en España la ley de autonomía del paciente (122) obliga a la práctica del $\mathrm{Cl}$, también en la psicosis. Reclama que se informe del procedimiento propuesto, sus resultados a corto y a largo plazo, sus efectos adversos, la existencia de tratamientos alternativos en la red pública 
o en la privada (1), permitiendo elegir y rechazar (122). Y cuando el paciente sea capaz debería poder contar con toda la información y poder decidir.

El Cl es una práctica que se está abriendo camino en otros países, entre ellos el Reino Unido, donde se defiende explícitamente desde instancias públicas el $\mathrm{Cl}$ y la autonomía del paciente psicótico (21). El Royal College of Psychiatrists anima a "apoyar a las personas en el logro de las metas que establecen para si mismas, aun si creyeran que las mismas no son realistas" (33), lo que podría incluir la opción de buscar otro camino de recuperación y, por ejemplo, vivir sin medicación.

Quizás no se pueda evitar la coerción en todos los casos, pero puede realizarse minimizando el daño: explicando más, sedando poco o no haciéndolo, mostrándose amable incluso en la contención, no dejando al paciente contenido, aislado y solo en una habitación. El paciente en crisis, incluso físicamente contenido, puede entender que se le considera, y es posible responder a la brusquedad con palabras en un tono suave y explicativo.

Es muy posible que adaptarse a estos requerimientos signifique cambiar no sólo el estilo asistencial actual sino también, en alguna medida, la estructura y funcionamiento de los centros y dispositivos de las Redes de Salud Mental, además de los servicios que deberían prestar.

La asistencia en urgencias psiquiátricas y unidades de hospitalización de agudos están orientados a la contención y bloqueo rápido de la sintomatología en situación de crisis. Aunque hay modelos alternativos de intervención, como Comunidades Terapéuticas, el Diálogo Abierto y otras $(21,98,100.123)$, que se han revelado capaces de contener la crisis, conseguir una mejor alianza terapéutica y ser menos traumáticos (52), y seguramente con mejores resultados a largo plazo.

\section{Conclusiones}

La práctica del consentimiento informado en personas con diagnóstico de psicosis, además de un imperativo ético y una obligación legal, es una buena práctica imprescindible para obtener la adhesión y participación del paciente en su proceso de recuperación, que puede traer mejores resultados a largo plazo

Un consentimiento informado de calidad supone informar en detalle de los objetivos, límites y posibles efectos adversos del tratamiento, de la existencia de tratamientos alternativos, y restituir el poder de decidir al paciente (125), quizás no en todos los momentos del proceso psicótico pero si como principio general.

Relacionarse con personas con un cuadro psicótico supone habilidades que es necesario entrenar para ejercer una práctica clínica correcta, y quienes se dedican a salud mental deberían formarse en ellas. Sin practicar esas habilidades hay poca justificación para la idea de que el paciente no es capaz de comprender y elegir. 
No es aceptable que la palabra del paciente psicótico deje de ser considerada, por transitar por dificultades intensas, máxime cuando hay procedimientos respetuosos que preservan su persona y consideran su estado coyuntural como una base para la integración y mejoría.

El paciente debe tener derecho a rehusar el tratamiento, especialmente cuando está en mantenimiento y no está incapacitado civilmente, y aun cuando se equivoque en su elección debe seguir siendo ayudado, como se hace en otras áreas sanitarias. Las divergencias entre el paciente y su entorno deben ser gestionadas por el clínico u otros profesionales, pero cuando el paciente sea capaz debería tener la última palabra.

La práctica clínica de tratar el proceso psicótico mediante fármacos puede ser adecuada para el paciente o la familia, pero puede no ser el tratamiento deseado para otros. De hecho tal práctica tiene efectos adversos y limitaciones cada vez más reconocidas desde el propio ámbito de la psiquiatría biológica

Los pacientes son muchas veces conscientes de sus dificultades, pero pueden creer que la ayuda que les presta la red asistencial les resulta dañina, y es posible que los servicios asistenciales, en su forma y filosofía asistencial actual, para muchos no sean adecuados $(11,15,21,25)$.

La hasta ahora tímida reacción de la psiquiatría biologicista ante los resultados mediocres de sus prácticas clínicas habituales (59) empieza a intuir la posibilidad de decidir del paciente psicótico (126), pero rara vez se justifica en el imperativo ético de restaurar su palabra silenciada. Sirva de ejemplo el importante estudio de seguimiento a 7 años de Wunderink y colaboradores, que podría impulsar para bien un cambio en los protocolos asistenciales, que afirma que los pacientes que rehusaron participar en su estudio, aproximadamente la mitad de candidatos, tenían un peor nivel de funcionamiento, menor adhesión a la terapia y más dificultades para comprometerse, sin que los autores tengan conciencia alguna de que seguramente no les satisfizo el tratamiento proporcionado (59).

Cuando se da la palabra a los pacientes, la defensa de su autonomía es una de sus principales reivindicaciones, como se observa en del movimiento de la recuperación ante lo que denominan "abuso de la psiquiatría" (Cuadro 3) $(20,81)$. Este es también un movimiento de autoayuda que cree en la recuperación, mediante una perspectiva clínica que trasciende los títulos académicos, basada en el apoyo y la comprensión, que obtiene resultados a considerar, y proporciona ciencia y debate desde su perspectiva de usuarios $(29,81)$.

Cuando los jueces dictan tratamientos forzosos pueden no conocer las alternativas y seguramente no imaginan que a largo plazo pueden empeorar al paciente, incluso de su propia psicosis. Si el paciente estuviera en situación de riesgo para si mismo o para otros se podría decidir de forma deliberativa lo qué hacer en cada situación concreta, considerando diversas alternativas clínicas Es posible que el paciente que no desee ser medicado pueda aceptar tener encuentros para hablar de sus preocupaciones en un entorno adecuado para obtener ayuda.

Resulta manifiesta la necesidad de investigar en profundidad tratamientos de diversos tipos sin subordinación al modelo actual dominante, además del alcance y las limitaciones de los antipsicóticos, tanto en la forma de uso actual como en la de hace unas décadas $(70,71)$. 
Desde un punto de vista asistencial debería disponerse en los próximos años de una variedad de servicios y dispositivos asistenciales para los pacientes psicóticos mayor que la actual, de forma que el consentimiento informado pueda realmente ejercerse, en lo que se refiere a optar por el tratamiento y el tipo de ayuda que el paciente considere más adecuada.

Seguramente no es un problema de coste económico. Recordemos que el antipsicótico depot de moda puede costar $500 €$ al mes (127), y con ello se podría dar ayuda psicosocial a dos o tres pacientes, o más. Además, mientras que los tratamientos de mantenimiento son a largo plazo, muchas veces indefinidos, el psicosocial suele establecer objetivos cronológicos de recuperación y reincorporación social, aunque puede que una parte de los pacientes no los consiga completamente o durante todo el tiempo de su proceso psicótico y su trayectoria vital.

\section{Referencias}

1. Moncrieff J. A straight talking introduction to psychiatric drugs. UK: PCCS Books, 2009.

2. Jackson G. Rethinking psychiatric drugs. A guide to informed consent. USA: AuthorHouse, 2005.

3. Jackson G. What Doctors May Not Tell You About Psychiatric Drugs. 2004. [Consultado el 1-82013] Disponible en: http://www.whale.to/drugs/Jackson.pdf

4. Luna Maldonado BE. El consentimiento informado en Psiquiatría. Estudio en los pacientes ambulatorios no psicóticos. Universidad de Cádiz, Tesis, 2003.

5. Moncrieff J. The Myth of the Chemical Cure: A Critique of Psychiatric Drug Treatment. 2Edit. USA: Palgrave Macmillan, 2009.

6. Healy D. Pharmageddon. USA: University of California Press, 2012.

7. Brown KB, Billcliff N, McCabe E. Informed consent to medication in long-term psychiatric inpatients. Psychiatric Bulletin, 25, 2001, p. 132-134.

8. Rutherford BR, Aizaga K, Sneed J, Roose SP. A survey of psychiatry residents' informed consent practices. J Clin Psychiatry. Apr; 68(4), 2007, p. 558-65.

9. Jain S, Lapid MI, Dunn LB, Roberts LW. Psychiatric Residents' Needs for Education About Informed Consent, Principles of Ethics and Professionalism, and Caring for Vulnerable Populations: Results of a Multisite Survey. Academic Psychiatry, 35:3, May-June, 2011, p. 184190.

10. Mauricio Espector E. Responsabilidad por mala práctica en Salud Mental Algunos puntos críticos Algunas reflexiones. Sinopsis, Marzo, año 23, no 43, 2007.

11. Davies J. Cracked: Why Psychiatry is Doing More Harm Than Good. UK: Icon Books, 2013.

12. Hall W. Discontinuación del Uso de Drogas Psiquiátricas: Una Guía Basada en la Reducción del Daño. The Icarus Project y Freedom Center, 2007 [Consultado el 1-8-2013] Disponible en: 
http://theicarusproject.net/downloads/GuiaReducciondelDanoDiscontinuaciondeDrogasPsiquiatr icas1EdOnline.pdf

13. Aminoff K, Boevink W, Daumerie N, Finkelstein C, Gauci D, Geoghegan P et al. Empoderamiento del usuario de salud mental. El empoderamiento no es un destino, sino un camino. Declaración de la Oficina Regional para Europa de la OMS Editado por Oficina Regional para Europa de la Organización Mundial de la Salud, 2010 [Consultado el 1-8-2013] Disponible en: http://www.msps.es/organizacion/sns/planCalidadSNS/pdf/Declaracion Empoderamiento OMS $\underline{\mathrm{pdf}}$

14. Oaks DW. MindFreedom International, en www.mindfreedom.org

15. Slade M. Personal recovery and mental illness. A guide for mental health professionals. UK: Cambridge University Press, 2009.

16. Weinstein J. (Editor) Mental Health service user involvement and recovery. UK: Jessica Kingsley Publishers, 2010.

17. Chamberlin J. On our own. Patient-controlled alternatives to the mental health system. USA: Hawthorn Books Inc, 1978.

18. T.A.I. (Tratamiento Ambulatorio Involuntario). Nuestras mentes, nuestras vidas. Palabras, reflexiones y alaridos contra el T.A.I. [Consultado el 1-8-2013] Accesible en: http://www.contralamedicacionforzosa.es/

19. Morrison A, Hutton P. Shiers D, Turkington D. Antipsychotics: is it time to introduce patient choice? BJP 201, 2012, 83-84.

20. Moncrieff J, Cohen D, Mason J. The patient's dilemma: an analysis of users' experiences of taking neuroleptics, en Coles S, Keenan S, Fiamond B. (Editors) Madness contested. Power and practice. UK: PCCS Books, 2013, p. 213-232.

21. Read J. Psychiatric drugs. Key issues and service user perspectives. UK: Palgrave, 2008.

22. Ruso J. Survivor-controlled research: a new foundation for thinking about psychiatry and mental health. FQS Forum: qualitative social research Vol 13, 1, 8, January 2012.

23. Gosden R. Punishing the patient. How psychiatrists misunderstand and mistreat schizophrenia. Australia: Melbourne Scribe Publications, 2001.

24. Kallert TW, Mezzich JE \& Monahan J. (Editors) Coercive treatment in psychiatry. UK: John Wiley \& sons, 2011.

25. Bentall R. Medicalizar la mente: ¿Sirven de algo los tratamientos psiquiátricos? Barcelona: Herder, 2011.

26. Read J, Mosher LR, Bentall R P (Editors). Modelos de locura: Aproximaciones psicológicas, sociales y biológicas a la esquizofenia. Barcelona: Herder, 2007. 
27. Brodwin P. Everyday ethics. Voices from the front line of community psychiatry. USA: University of California Press, 2013.

28. Bellack AS. Modelos científicos y de usuarios sobre la recuperación en la esquizofrenia: coincidencias, contrastes e implicaciones. Schizophrenia Bulletin (Ed Esp), 2(3), 2007, p. 117128.

29. Beresford P. A straight talking introduction to being a mental health service user. UK: PCCS Books, 2010.

30. Dellar R, Curtis T, Leslie E. (Editors) Mad Pride. UK: Chipmunka Publishing, 2000.

31. Hearing Voices Network en www.hearing-voices.org

32. Pinals DA. Informed consent: Is your patient competent to refuse treatment? Current Psychiatry Vol. 8, No. 4, 2009.

33. The Royal College of Psychiatrists. Fair Deal for mental health manifesto. [Consultado 1-82013] Disponible en: http://bjp.rcpsych.org/content/197/1/1.full.pdf

34. Couceiro A. (Editor) Bioética para clínicos. Esp.: Triacastela, 1999.

35. Baca E, Lázaro J. (Editors) Hechos y valores en psiquiatría. Esp: Triacastela, 2002.

36. Bloch S, Chodoff P, Green SA (Editors) La ética en psiquiatría. Esp.: Triacastela, 2002.

37. Amador X. !No estoy enfermo, no necesito ayuda! Esp.: Mayo Ediciones, 2010.

38. Andreasen N. The broken brain. USA: Harper \& Row, 1984.

39. Cuesta MJ, García de Jalón E, Campos MS, Ibáñez B, Sánchez-Torres AM, Peralta V. Duration of untreated negative and positive symptoms of psychosis and cognitive impairment in first episode psychosis. Schizophrenia Research 141, 2012, p. 222-227.

40. Stahl SM. Psicofarmacología esencial: bases neurocientíficas y aplicaciones clínicas. Esp.: Ariel, 2002.

41. Chinchilla Moreno A. (Editor) Las esquizofrenias: sus hechos y valores clínicos terapéuticos. Esp.: Masson, 2007.

42. Bobes J, Sainz J. (Editors) El impacto social de la esquizofrenia. Esp.: Glosa, 2013.

43. Vallejo Ruiloba J. (Editor). Introducción a la psicopatología y la psiquiatría. 7ํㅡㄹ Ed. Esp.: Masson, 2011.

44. Andreasen N. Un cerebro feliz. La conquista de la enfermedad mental en la era del genoma. Esp.: Ars Medica, 2003.

45. Berrios G, Fuentenebro F. Delirio: Historia, Clínica, Metateoría. Esp.: Trotta, 1996.

46. Sáiz Ruiz J. Esquizofrenia: Enfermedad del cerebro y reto social. Esp.: Masson, 1999.

47. Geekie J, Read J. El sentido de la locura: La exploración del significado de la esquizofrenia. Barcelona: Herder, 2012. 
48. Cavieres A. ¿Perdida de la conciencia de enfermedad o percepción subjetiva en personas enfermas de esquizofrenia? Rev GPU 9;1: 2013, p. 59-63.

49. Watters E. Crazy Like Us: The Globalization of The American Psyche. USA: Free Press, 2010.

50. Chadwick P. Terapia cognitiva basada en la persona para la psicosis perturbadora. Esp.: Fundación para la investigación y el tratamiento de la esquizofrenia y otras psicosis, 2009.

51. Boyle M. Todo es hecho con humo y espejos. O, de como crear la ilusión de que la esquizofrenia es una enfermedad cerebral. INTECO Instituto de Terapia Cognitiva, Santiago de Chile 2002, [Consultado 1-8-2013] Disponible en: http://www.inteco.cl/articulos/020/esquizofrenia.pdf

52. Seikkula J, Olson M E. El diálogo abierto como procedimiento de trabajo en la psicosis aguda: su "poética" y "micropolítica". Revista de psicoterapia Vol XVI, 63/53, 2005, p. 135-154.

53. Fuller Torrey E. Superar la esquizofrenia. Esp.: Planeta, 2006.

54. Alanen Y. La esquizofrenia. Sus orígenes y su tratamiento adaptado a las necesidades del paciente. Esp.: Fundación para la investigación y el tratamiento de la esquizofrenia y otras psicosis, 2003.

55. Seikkula J, Alakare B, Aaltonen J. The Comprehensive Open-Dialogue Approach in Western Lapland: II. Long-term stability of acute psychosis outcomes in advanced community care. Psychosis, 3, 2011, p. 192-204.

56. Candia P C, Barba A C. Mental capacity and consent to treatment in psychiatric patients: the state of research. Current Opinion in Psychiatry 24, 2011, p. 442-446.

57. Oaks DM. Whose Voices Should Be Heard? The Role of Mental Health Consumers, Psychiatric Survivors, and Families, en Dudley M, Silove D, Gale F.(Editors) Mental Health and Human Rights vision, praxis, and courage. USA: Oxford University Press, 2012, p. 566-77.

58. Wunderink L, Nieboer RM, Wiersma D, Sytema S, Nienhuis FJ. Recovery in Remitted FirstEpisode Psychosis at 7 Years of Follow-up of an Early Dose Reduction/Discontinuation or Maintenance Treatment Strategy: Long-term Follow-up of a 2-Year Randomized Clinical Trial. JAMA Psychiatry. Sep 1;70(9), 2013 p. 913-20.

59. McGorry P, Alvarez-Jimenez M, Killackey E. Antipsychotics in First-Episode Psychosis: Less Is More. JAMA Psychiatry. 70(9): 2013, p. 898-900.

60. Fontela E, Amboage MT, Ávila J, González S, Vázquez C, Arias DM. Características de la politerapia con antipsicóticos en una muestra de pacientes con esquizofrenia a seguimiento en una unidad de salud mental. Ponencias y pósters del XII Congreso Nacional de Psiquiatría, Valencia, 2008. [Consultado 1-8-2013] Disponible en: http://www.sepsiq.org/ psiquiatriavalencia08/programa3 posters.php@posters=23

61. Arilla JE, Fernández ME, Viñuales E, Lozano I. Politerapia antipsicótica en una unidad de hospitalización Psiquiátrica. Rev Psiquiatr Salud Ment. 3(3), 2010, p. 90-96. 
62. Landa Contreras E, Fortes Álvarez JL. Monoterapia versus Politerapia en el tratamiento de la Psicosis: ¿Con qué nos quedamos? Rev Clín Med Fam. 4 (2): 2011, p. 140-145.

63. Edwards J, McGorry PD. La intervención precoz en la psicosis: Guía para la creación de servicios de intervención precoz en la psicosis. Madrid: Fundación para la investigación y el tratamiento de la esquizofrenia y otras psicosis, 2004.

64. Scott T. America Fooled: The Truth About Antidepressants, Antipsychotics And How We've Been Deceived. USA: Argo Publishing, 2006.

65. Kozma CM, Saltón T, Dirani R, Fastenau J, Gopal, S, Hough D. Cambios en las hospitalizaciones y las visitas al servicio de urgencias en pacientes con esquizofrenia que reciben palmitato de paliperidona: resultados de un ensayo clínico con una fase de extensión abierta de 52 semanas. Curr Med Res Opin. 27(8), 2011, 603-11 (Traducción y texto en español ofrecido por Janssen).

66. Rebolledo Moller S, Lobato Rodríguez MJ. Cómo afrontar la esquizofrenia: Una guía para familiares, cuidadores y personas afectadas. Madrid: Aula Médica, 2005.

67. Valenstein M, Ganoczy D, McCarthy JF, Myra Kim H, Lee TA, Blow FC. Antipsychotic adherence over time among patients receiving treatment for schizophrenia: a retrospective review. J Clin Psychiatry. Oct;67(10): 2006, p. 1542-50.

68. Goff DC, Hill M, Freudenreich O. Strategies for improving treatment adherence in schizophrenia and schizoaffective disorder. J Clin Psychiatry. 71 Suppl 2: 1010, p. 20-6.

69. Valenstein M, Blow FC, Copeland LA, McCarthy JF, Zeber JE, Gillon L \& al. Poor antipsychotic adherence among patients with schizophrenia: medication and patient factors. Schizophr Bull. 30(2): 2004, 255-64.

70. May PR. Esquizofrenia: revisión de métodos terapéuticos, en Freedman AM, Kaplan HI, Sadock BJ. (Editors) Tratado de psiquiatría, Vol 1, Esp. Salvat, 1982, p. 1031-48.

71. May PR. Esquizofrenia: evaluación de métodos terapéuticos, en Freedman AM, Kaplan HI, Sadock BJ. (Editors) Tratado de psiquiatría. Vol 1, Esp. Salvat, 1982, p. 1067-97.

72. Osborn DP, Levy G, Nazareth I, Petersen I, Islam A, King MB. Relative risk of cardiovascular and cancer mortality in people with severe mental illness from the United Kingdom's General Practice Rsearch Database. Arch Gen Psychiatry. Feb;64(2): 2007, p. 242-9.

73. Ho BC, Andreasen NC, Ziebell S, Pierson R, Magnotta V. Long-term Antipsychotic Treatment and Brain Volumes A Longitudinal Study of First-Episode Schizophrenia. Arch Gen Psychiatry. 68(2), 2011, p. 128-137.

74. Moncrieff J, Leo J. A systematic review of the effects of antipsychotic drugs on brain volume. Psychol Med. Sep;40(9) 2010, 1409-22.

75. Jackson G. Drug-Induced Dementia: a perfect crime. USA: AuthorHouse, 2009. 
76. Molina V. Parámetros indicativos de neurodegeneración. Revista Mente y Cerebro 20: 2006, p. 80-3

77. Healy D, Harris M, Tranter R, Gutting P, Austin R, Jones-Edwards G et al. Lifetime suicide rates in treated schizophrenia: 1875-1924 and 1994-1998 cohorts compared. Br J Psych. Mar;188, 2006, p. 223-8.

78. Weinmann S, Aderhold V. Antipsychotic medication, mortality and neurodegeneration: The need for more selective use and lower doses. Psychosis 2, (1): 2009, p. 50-69.

79. Frances A. Saving normal. USA: Harper Collins, 2013.

80. Saha S, Chant D, McGrath J. A systematic review of mortality in schizophrenia: is the differential mortality gap worsening over time? Arch Gen Psychiatry. Oct;64(10), 2007, p. 112331.

81. Sweeney A, Beresford P, Faulkner A, Nettle M, Rosse D. This is survivor research. UK: PCCS Books, 2009.

82. Ciompi L. Afecto-Logica. el Vínculo Entre el Afecto y la Lógica. una Contribución al Estudio de la Esquizofrenia. Esp.: Fundación para la investigación y el tratamiento de la esquizofrenia y otras psicosis, 2010.

83. Harrow, M., Jobe T.H. Factors involved in outcome and recovery in schizophrenia patients not on antipsychotic medications: a 15-year multifollow-up study The Journal of nervous and mental disease 195, (5) p. 2007,406-14.

84. Helgason L. Twenty years' follow-up of first psychiatric presentation for schizophrenia. Acta Psychiatrica Scandinavia, 81, 1990, 231-235.

85. Hegarty JD, Baldessarini RJ, Tohen M, Waternaux C, Oepen G. One hundred years of schizophrenia: a meta-analysis of the outcome literature. Am J Psychiatry. Oct;151(10), 1994, p. 1409-16.

86. Jin H, Shih PA, Golshan S, Mudaliar S, Henry R, Glorioso DK \& AL. Comparison of longer-term safety and effectiveness of 4 atypical antipsychotics in patients over age 40: a trial using equipoise-stratified randomization. J Clin Psychiatry. Jan;74(1): 2013, p. 10-8.

87. Whitaker R. Anatomy of an Epidemic: Magic Bullets, Psychiatric Drugs, and the Astonishing Rise of Mental Illness in America. USA: Crown, 2010.

88. Harrow M, Jobe TH, Faull RN. Do all schizophrenia patients need antipsychotic treatment continuously throughout their lifetime? A 20-year longitudinal study. Psychol Med.Oct;42(10) 2012, p. 2145-55.

89. Goldacre B. Mala farma. Cómo las empresas farmacéuticas engañan a los médicos y perjudican a los pacientes. Esp.: Paidós, 2013. 
90. Cosgrove L, Krimsky S. A Comparison of DSM-IV and DSM-5 Panel Members' Financial Associations with Industry: A Pernicious Problem Persists. PLoS Med. 9 (3): e1001190. doi:10.1371/ journal.pmed.1001190, 2012.

91. García-Martínez J. Técnicas narrativas en psicoterapia. Esp.; Síntesis, 2012.

92. Rhodes J, Jakes S. Narrative CBT for Psychosis. UK: Routledge Chapman \& Hall, 2009.

93. Marley JA. Family involvement in treating schizophrenia. Models, essentials skills, and process. USA: Haworth Clinical Press, 2004.

94. Lobban F, Barrowclough C. (Editors) A casebook of family interventions for psychosis. USA: Wiley-Blackwell, 2009.

95. Garfield D, Mackler D. Beyond Medication: Therapeutic Engagement and the Recovery from Psychosis, UK: Routledge, 2008.

96. Morrison A, Renton J C, Dunn H, Bentall R. Terapia cognitiva de la psicosis: Un enfoque basado en la formulación. Esp.: Herder, 2012.

97. Morris E M, Johns L C, Oliver J E. (Editors). Acceptance and commitment therapy and mindfulness for psychosis. UK: Wiley-Blackwell, 2013.

98. Heath DS. Home treatment for acute mental disorders. USA: Bruner Routledge, 2005.

99. Berke JH, Fagan M, Mak-Pearce G, Pierides-Muller S. Beyond madness. PsychoSocial interventions in psychosis. Jessica Kingsley Publishers, UK, 2002.

100. Seikkula J, Alakare B, Aaltonen J. El enfoque del dialogo abierto. Principios y primeros resultados de investigación sobre un episodio psicótico. Sistemas familiares 17 (2) 2001, p. 75 87.

101. Romme M, Escher S. Dando sentido a las voces. Esp.: Fundación para la investigación y el tratamiento de la esquizofrenia y otras psicosis, 2005.

102. Longden E, Cornstens D, Dillon J. Recovery, discovery and revolution: The work of Intervoice and the Hearing Voices Movement. en Coles S, Keenan S, Fiamond B. Madness contested. Power and practice. PCCS Books, UK, 2013, 161-180.

103. Corstens D, Longden E, Rydinger B, Bentall R, \& van Os J. Treatment of hallucinations: $A$ comment. Psychosis, 5:1, 2013, 98-102.

104. Karon BP. The tragedy of schizophrenia without psychotherapy. J Am Acad Psychoanal Dyn Psychiatry. Spring;31(1) 2003, p. 89-118.

105. Morrison AP, French P, Walford L, Lewis SW, Kilcommons A, Green J, Parker S, Bentall RP. Cognitive therapy for the prevention of psychosis in people at ultra-high risk: randomised controlled trial. Br J Psychiatry. Oct;185: 2004, p. 291-7.

106. Warner R. Recovery from schizophrenia. Psychiatry and political economy. USA: Bruner Routledge, 2004. 
107. Karon BP, Vandenbos GR. Psychoteraphy of schizophrenia. The treatment of choice. USA: Aronson, 1981.

108. Seikkula J, Aaltonen J, Alakare B, Haarakangas K, Kera"Nen J, Lehtinen K. Five-year experience of first-episode nonaffective psychosis in open-dialogue approach. Psychotherapy Research 16, 2006, 214-28.

109. Grant A, Biley F, Walker H. (Editors) Our encounters with madness. UK: PCCS Books, 2011.

110. Coles S, Keenan S, Fiamond B. Madness contested. Power and practice. UK: PCCS Books, 2013.

111. Shaw B. Peer Support, en Coles S, Keenan S, Fiamond B. Madness contested. Power and practice. UK: PCCS Books, 2013, p. 293-306.

112. Stastny P, Lehmann P. Alternatives Beyond Psychiatry. Alemania: Peter Lehmann Publishing, 2007.

113. Romme M, Escher S, Dillon J, Corstens D, Morris M. (Editors) Living with Voices: 50 Stories of Recovery. UK: PCCS Books, 2009.

114. Williams P. Rethinking Madness: Towards a Paradigm Shift in Our Understanding and Treatment of Psychosis. USA: Sky's Edge Publishing, 2012.

115. Warner R. Does the scientific evidence support the recovery model? The Psychiatrist, 34, 2010, 3-5.

116. Davidson L, Harding C, Spaniol L. (Editors) Recovery from Severe Mental Illnesses: Research Evidence And Implications for Practice, Volume 1 \& 2. USA: Center for Psychiatric Rehabilitation, Boston Univ, 2005.

117. Williams P. Full Recovery from Schizophrenia? 2012 [Consultado 1-8-2013] Disponible en: http://behindthelockeddoors.wordpress.com/2012/07/02/full-recovery-from-schizophrenia/

118. Seale C, Chaplin R, Lelliott P, Quirk A. Antipsychotic medication, sedation and mental clouding: An observational study of psychiatric consultations. Soc Sci Med. Aug;65(4): 2007, p. 698-711.

119. Moncrieff J, Cohen D, Mason JP. The subjective experience of taking antipsychotic medication: a content analysis of Internet data. Acta Psychiatr Scand. Aug;120(2): 2009, p. 102-11.

120. Declaración del grupo Mad Pride.[Consultado 1-8-2013] Disponible en: http://www.theicarusproject.net/downloads/GuiaReducciondelDanoDiscontinuaciondeDrogasPsi quiatricas1EdOnline.pdf

121. Russo J. Survivor-controlled research: $A$ new foundation for thinking about psychiatry and mental health. Forum: qualitative social research, FQS. Vol 13, no1, Art 8, January 2012.

122. Ley de autonomía del paciente, Ley 41/2002 de 14 de noviembre 2002.

123. Mackler D. Healing homes. DVD, 2011. 
124. American Diabetes Association. Consensus Development Conference on Antipsychotic Drugs and Obesity and Diabetes. Diabetes Care, Volume 27, Number 2, February 2004, p. 596-601.

125. Valverde MA, Inchauspe JA. El Consentimiento Informado como proceso en Salud Mental. Una propuesta para el caso TDA/H. En revisión e Cuadernos de Bioética, 2013.

126. van Os J, Howes OD. Antipsychotic drugs for prevention of relapse. Lancet. Jun 2;379(9831): 2012, 2030-1.

127. Valverde MA Curar con lo último: el palmitato de paliperidona. Rev. Norte de salud mental, vol. XI, no 46: 2013, p. 59-74.

128. Morrison A, Hutton P, Wardle M, Spencer H, Barratt S, A. Brabban A et al. Cognitive therapy for people with a schizophrenia spectrum diagnosis not taking antipsychotic medication: an exploratory trial. Psychol Med. May;42(5): 2012, p. 1049-56.

129. Priebe S, Burns T, Craig TK. The future of academic psychiatry may be social. BJP May 202 2013, p. 319-320.

Fecha de recepción: 26 de septiembre de 2013

Fecha de aceptación: 15 de octubre de 2013 